\title{
Oncocitoma renal: tem a URO-TC utilidade no diagnóstico histológico?
}

\author{
João Almeida Dores ${ }^{a, *}$, Peter Kronenberg ${ }^{a}$, Pedro Bargão Santos ${ }^{a}$, \\ Sérgio Ferreira ${ }^{\mathrm{b}}$ e Francisco Carrasquinho Gomes ${ }^{\mathrm{a}}$
}

\author{
a Serviço de Urologia, Hospital Prof. Doutor Fernando Fonseca, Amadora, Portugal \\ b Serviço de Imagiologia, Hospital Prof. Doutor Fernando Fonseca, Amadora, Portugal
}

Recebido a 15 de março de 2016; aceite a 21 de junho de 2016

Disponível na Internet a 22 de julho de 2016

\author{
PALAVRAS-CHAVE \\ Oncocitoma renal; \\ Carcinoma de células \\ renais; \\ Diagnóstico; \\ URO-TC
}

\begin{abstract}
Resumo
Introdução: Ao longo dos últimos anos, a crescente utilização de exames imagiológicos, nomeadamente ecografia e tomografia computorizada (TC), traduziu-se num aumento do diagnóstico incidental de tumores renais, sobretudo pequenas massas renais $(<4 \mathrm{~cm})$. 0 conhecimento de que até $30 \%$ destas massas podem ser benignas, entre elas os oncocitomas, levou à procura de métodos de diagnóstico mais eficazes, de forma a evitar situações de sobretratamento e de forma a tomaram-se decisões terapêuticas mais fundamentadas.

Objetivos: Analisar retrospetivamente uma série de tumores renais histologicamente comprovados, nomeadamente oncocitomas e carcinoma de células renais (CCR), e verificar se existem diferenças morfológicas e/ou nos padrões de captação de contraste através da URO-TC.

Material e métodos: Identificámos todos os tumores renais entre 2004-2015 com o diagnóstico histológico de oncocitoma e de CCR. Estes resultados foram obtidos por biopsia do tumor renal, tumorectomia/nefrectomia parcial ou nefrectomia radical. Registámos e comparámos as características morfológicas e os padrões de captação de contraste na fase nefrográfica com medição de unidades de Hounsfield (HU) dos oncocitomas e dos CCR (células claras), selecionados de acordo com a dimensão (aprox. idêntica à dos oncocitomas) e obtidos na sequência de tumorectomia renal ou nefrectomia radical.

Resultados: Identificaram-se 16 CCR e 31 oncocitomas, dos quais 15 foram excluídos por não termos acesso às imagens de TC no sistema informático. A dimensão média dos oncocitomas foi $3,7 \mathrm{~cm}(1,8-14)$ e a dos CCR $3,5 \mathrm{~cm}(1,9-8,4)$. A atenuação de contraste média dos oncocitomas e dos CCR na fase sem contraste foi de $33 \mathrm{HU}$ e $32 \mathrm{HU}$, respetivamente. Na fase nefrográfica, a captação média de contraste para os oncocitomas foi de $47,5 \mathrm{HU}$ e $47,4 \mathrm{HU}$ para os CCR. $\mathrm{Na}$ fase nefrográfica, a diferença de atenuação entre os oncocitomas e o parênquima renal normal foi $43,5 \mathrm{HU}$ e a diferença de atenuação entre os CCR e o parênquima renal normal foi $59,7 \mathrm{HU}$. Estes resultados foram estatisticamente significativos $(p<0,05)$. Não se identificaram outras alterações na fase excretora da TC, nem diferenças relevantes de carácter morfológico, nomeadamente nos contornos das lesões, presença de calcificações, ou de cicatriz central.
\end{abstract}

\footnotetext{
* Autor para correspondência.

Correio eletrónico: Joaoalmeidadores@gmail.com (J. Almeida Dores).
} 
Conclusões: Na avaliação imagiológica por URO-TC, nomeadamente na fase nefrográfica, parece existir uma tendência para maior isodensidade dos oncocitomas em relação ao parênquima renal normal. Este achado poderá contribuir para uma melhor decisão terapêutica, na medida em que nos pode direcionar para uma biopsia de confirmação em detrimento da excisão cirúrgica. (c) 2016 Associação Portuguesa de Urologia. Publicado por Elsevier España, S.L.U. Este é um artigo Open Access sob uma licença CC BY-NC-ND (http://creativecommons.org/licenses/bync-nd/4.0/).

\section{KEYWORDS}

Renal oncocytoma; Renal cell carcinoma; Diagnosis; URO-CT

\section{Renal oncocytoma: Is URO-CT useful in histological diagnosis?}

\begin{abstract}
Introduction: Over the past few years, the increasing use of cross-sectional imaging, including ultrasound and computed tomography imaging, resulted in an increase incidental diagnosis of renal tumors, especially small renal masses $(<4 \mathrm{~cm})$. The knowledge that $30 \%$ of these masses may be benign, including oncocytomas led to the investigation for more effective methods of diagnosis in order to avoid overtreatment situations.

Objectives: The authors decided to analyse and compare contrast enhancement patterns of oncocytomas and clear-cell renal cell carcinoma (ccRCC) to predict histology.

Material and methods: Between 2004-2015 we retrospectively identified 32 patients with either histological confirmation of renal oncocytoma $(N=16)$ or $\operatorname{ccRCC}(N=16)$ who underwent percutaneous biopsy, total or partial nephrectomy. The relative attenuation of solid renal lesions and normal renal cortex was determined in the unenhanced and nephrographic phase. Statistical comparison was carried out by Mann-Withney test.

Results: The oncocytomas and cc-RCC average size was $3.7 \mathrm{~cm}$ [1.8 to 14 ] and $3.5 \mathrm{~cm}$ [1.9 to 8.4], respectively. The average attenuation in the unenhanced phase was $33 \mathrm{HU}$ and $32 \mathrm{HU}$, respectively. In nephrographic phase, the average contrast enhancement was 47.5 and $47.4 \mathrm{H}$, respectively. In nephrographic phase, the attenuation difference between the oncocytomas and normal renal cortex was $43.5 \mathrm{HU}$ and the attenuation difference between the cc-RCC and normal renal cortex was $59.7 \mathrm{HU}$. These results were statistically significant $(p<0.05)$.

Conclusions: In the nephrographic phase, URO-CT reveals that oncocytomas have greater isodensity to the normal renal cortex compared to cc-RCC. This finding can help us to determine which lesions we should biopsy or not.

(c) 2016 Associação Portuguesa de Urologia. Published by Elsevier España, S.L.U. This is an open access article under the CC BY-NC-ND license (http: / / creativecommons.org/licenses/by-nc-nd/ $4.0 /)$.
\end{abstract}

\section{Introdução}

Atualmente, cerca de $66 \%$ dos tumores renais são diagnosticados incidentalmente ${ }^{1}$ e definidos como pequenas massas renais (PMR), ou seja, massas sólidas ou parcialmente sólidas, com dimensão igual ou inferior a $4 \mathrm{~cm}$ e que captam contraste na tomografia computorizada (TC) ou ressonância magnética (RMN), sendo consideradas suspeitas para carcinoma de células renais $(C C R)^{2}$. Paralelamente, estudos sugerem que a verdadeira benignidade histológica das PMR é superior ao anteriormente pensado, entre os $20-30 \%^{3-7}$. Mesmo nos casos de malignidade, só $20-25 \%$ destas massas apresentam características histopatológicas agressivas e potencialmente letais ${ }^{3}$. Entre as massas sólidas benignas mais frequentes, encontram-se os oncocitomas. À semelhança dos CCR do tipo cromófobo derivam histologicamente das células intercaladas do ducto coletor, no entanto, são genotipicamente distintos ${ }^{8}$. Clinica e imagiologicamente, o diagnóstico diferencial com CCR continua a ser um verdadeiro dilema para o urologista. Clinicamente e em ambos os casos, o diagnóstico é frequentemente incidental e em doentes assintomáticos; o pico de incidência verifica-se aos 70 anos de idade e com uma predominância no sexo masculino de $2: 1^{9}$.

Imagiologicamente (URO-TC), o oncocitoma caracteriza-se como uma lesão bem definida, com contornos regulares e com padrão homogéneo de captação de contraste ${ }^{10,11}$. A típica cicatriz central, frequentemente associada aos oncocitomas de grandes dimensões, não pode ser diferenciada da necrose central dos CCR e, por isso, a sua presença constitui um valor preditivo diagnóstico muito baixo ${ }^{12}$.

À semelhança do que se verifica noutras áreas da oncologia urológica, os diagnósticos fazem-se em idades cada vez mais avançadas (> 75anos), em doentes com múltiplas comorbilidades e, em muitos casos (até 25\%), em doentes com insuficiência renal crónica (IRC). De forma a combater o sobretratamento e as indicações cirúrgicas com base em «probabilidades», tem-se assistido a uma maior aceitação e realização de biopsias de tumores renais e tem-se procurado desenvolver novas técnicas de imagem, nomeadamente na RMN multiparamétrica, no entanto, ainda sem resultados conclusivos $^{13}$. 
Tendo em conta os dados supracitados e as constantes restrições económicas no campo da saúde, consideramos que os progressos devem ser feitos no campo do diagnóstico de imagem, com os recursos que já temos atualmente à disposição, nomeadamente por TC. Este trabalho teve como objetivo avaliar retrospetivamente se existem diferenças no estudo por TC, na morfologia e principalmente nos padrões de atenuação de contraste entre as 2 entidades histológicas malignas e benignas mais frequentes, o CCR (células claras [CCR-CC]) e o oncocitoma, respetivamente.

\section{Material e métodos}

Analisámos retrospetivamente a histologia de todos os tumores renais entre 2005-2013 submetidos a biopsia percutânea, tumorectomia/nefrectomia parcial e nefrectomia radical. Identificámos 31 oncocitomas. Destes, excluímos 15 por não termos acesso aos exames de imagem, perfazendo um total de 16 oncocitomas. Selecionámos 16 CCR, tipo CCR-cC, com dimensão média semelhante à dos oncocitomas.

Foram realizados exames no aparelho GE LightSpeed Plus 4 Slice CT da GE Medical Systems - Advantage Workstation 4.0 Ultra 60, com helical thickness de $5 \mathrm{~mm}$, beam collimation deg $10 \mathrm{~mm}$, pitch de 1,5:1 e detector configuration de $4 \times 1,25$. Foi usado contraste EV não-iónico (Ultravist 300).

Para todas as lesões avaliámos as características morfológicas, nomeadamente, dimensão, densidade (unidades de Hounsfield [HU]), contornos, presença de calcificações, presença de cicatriz central, presença de trombose da veia renal e secundarismo, na fase sem contraste e fase nefrográfica.

Após a administração do contraste, a avaliação da atenuação em HU foi feita sempre na fase nefrográfica, aos 90 segundos após a administração de contraste.

Para ambas as entidades (oncocitoma vs. CCR-CC) desenharam-se as áreas circulares de interesse (ROI) quer no tumor quer no córtex renal (fig. 1). Na fase nefrográfica determinou-se o valor médio de atenuação de contraste em HU para cada tumor, no corte de maior diâmetro. 0

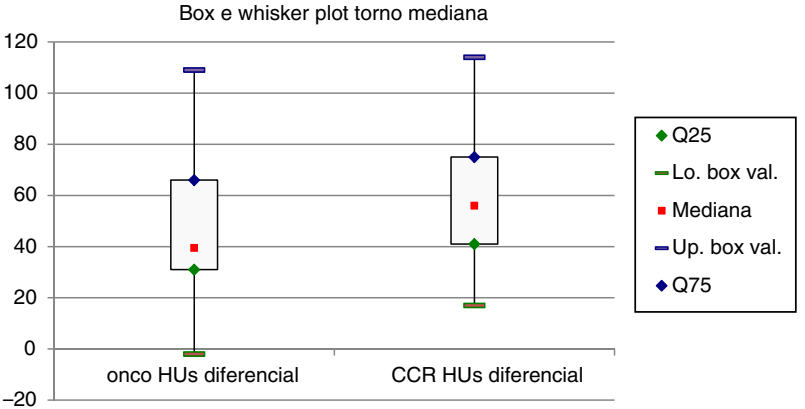

Figura 2 Box e Whisker plots dos ratios de atenuação entre o córtex renal e a lesão tumoral na fase nefrográfica. As caixas representam a amplitude de atenuação entre o percentil 25 ao percentil 75. 0 ponto vermelho representa as medianas e as barras horizontais de maior espessura os extremos.

valor médio de atenuação do córtex renal foi determinado pelo cálculo médio entre 2 pontos diferentes com um corte de diâmetro aproximado de $0,5 \mathrm{~cm}^{2}$. Para todos os casos, determinou-se o diferencial de atenuação contraste entre 0 córtex renal e o tumor (HU do córtex - HU do tumor).

Para a análise estatística, utilizou-se o teste de Mann-Whitney. Consideraram-se estatisticamente significativos valores de $p$-value $<0,05$ (fig. 2).

\section{Resultados}

A idade média dos doentes com oncocitoma renal foi de 62 anos (41-79; 8 sexo masculino, 8 sexo feminino) e a dos doentes com CCR foi de 57 anos (32-76; 12 sexo masculino, 6 sexo feminino). A dimensão média dos oncocitomas foi $3,7 \mathrm{~cm}(1,8-14)$ e a dos CCR $3,5 \mathrm{~cm}(1,9-8,4)$. Dos 16 oncocitomas, 7 estavam localizados no rim direito, 9 no rim esquerdo. Dos 16 CCR, 6 localizados no rim direito, 10 no rim esquerdo.

Em termos morfológicos todas as lesões eram homogéneas, sem extensas áreas de necrose. Não se verificou nenhum caso de cicatriz central (tabela 1).

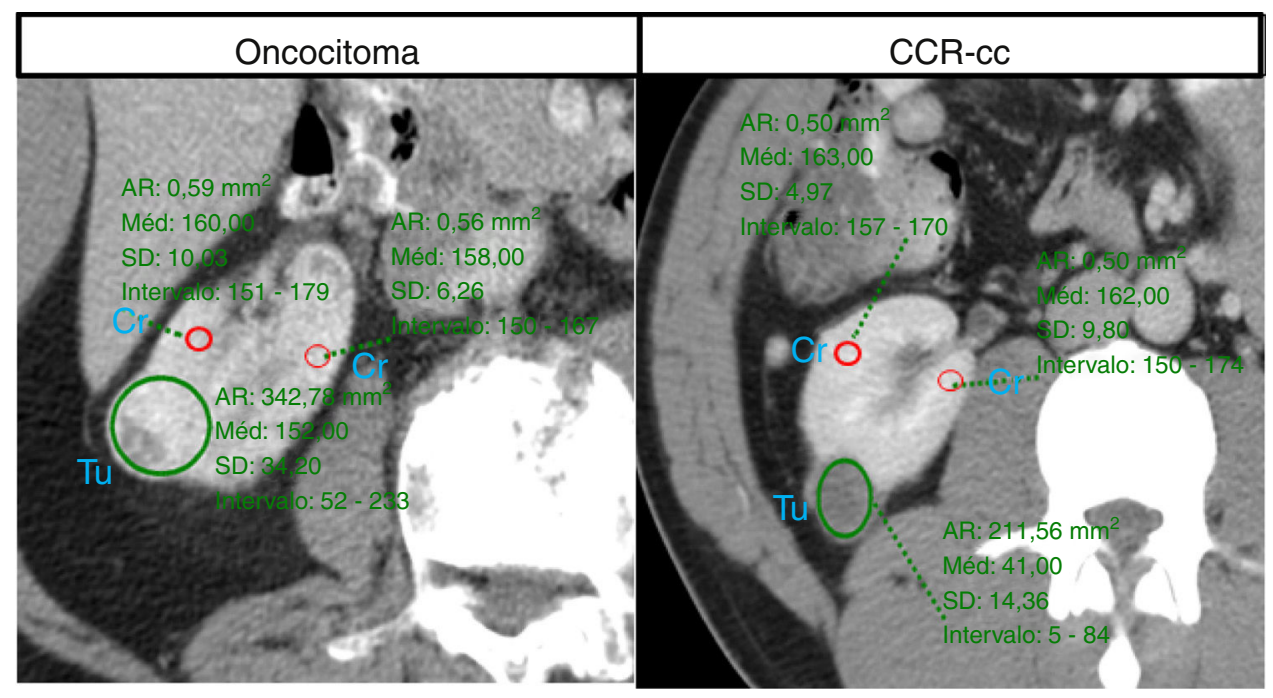

Figura 1 Determinação da atenuação de contraste em unidades de Hounsfield no tumor (Tu) e em 2 pontos diferentes medidos no córtex renal (cr). 
Tabela 1 Avaliação das características morfológicas

\begin{tabular}{|c|c|c|c|c|c|c|}
\hline & \multicolumn{2}{|c|}{ Contornos } & \multirow[t]{2}{*}{ Calcificações } & \multirow{2}{*}{$\begin{array}{l}\text { Cicatriz } \\
\text { central }\end{array}$} & \multirow{2}{*}{$\begin{array}{l}\text { Trombose da } \\
\text { veia renal }\end{array}$} & \multirow[t]{2}{*}{ Secundarismo } \\
\hline & Regulares & Irregulares & & & & \\
\hline Oncocitoma & 14 & 2 & 0 & 0 & 0 & 0 \\
\hline CCR & 7 & 9 & 2 & 0 & 0 & 0 \\
\hline
\end{tabular}

Tabela 2 Determinação da densidade, captação de contraste e do diferencial de atenuação de contraste entre córtex renal e o tumor

\begin{tabular}{|c|c|c|c|}
\hline & $\begin{array}{l}\text { Fase sem contraste } \\
\text { média }(\mathrm{HU})\end{array}$ & Fase nefrográfica & \\
\hline & & $\begin{array}{l}\text { Captação de } \\
\text { contraste média (HU) }\end{array}$ & $\begin{array}{l}\text { (HU) córtex- (HU) } \\
\text { tumor média }\end{array}$ \\
\hline Oncocitoma & 33 & 47,5 & 43,5 \\
\hline \multirow[t]{2}{*}{ CCR } & 32 & 47,4 & 59,7 \\
\hline & \multicolumn{2}{|c|}{ Sem diferença estatisticamente significativa } & $\mathrm{p}<0,05$ \\
\hline
\end{tabular}

HU: unidades de Hounsfield.

$\mathrm{Na}$ fase sem contraste (tabela 2), a atenuação de contraste média dos oncocitomas e dos CCR foi de $33 \mathrm{HU}$ e $32 \mathrm{HU}$, respetivamente. Na fase nefrográfica, a captação média de contraste para os oncocitomas e dos CCR foi de 47,5 HU e 47,4 HU, respetivamente.

$\mathrm{Na}$ fase nefrográfica, a diferença de atenuação de contraste entre os oncocitomas e o parênquima renal normal foi 43,5 HU e a diferença de atenuação entre os CCR e o parênquima renal normal foi 59,7 HU. Estes resultados foram estatisticamente significativos $(p<0,05)$.

\section{Discussão}

Os resultados obtidos relativamente às características morfológicas (contornos, calcificações, cicatriz central) de ambos tumores (oncocitoma vs. CCR-cc) não revelaram qualquer diferença que seja relevante para a sua distinção. A presença de cicatriz central descrita na literatura como presente em cerca de $33 \%$ dos oncocitomas ${ }^{14}$, não se verificou em nenhum dos casos. Uma explicação poderá estar no facto de, em todo o estudo, termos incluído quase exclusivamente tumores de pequenas dimensões $(<5 \mathrm{~cm})$, sendo esta característica típica de oncocitomas de grandes dimensões.

Em termos de densidade e captação de contraste também não identificámos diferenças com significado estatístico (fig. 3). A densidade e captação de contraste dos oncocitomas e CCR-cc são aproximadamente idênticas, resultados que vão ao encontro de outros estudos anteriormente realizados ${ }^{15}$.

No entanto, ao compararmos a diferença de atenuação de contraste entre o córtex renal e os tumores na fase nefrográfica, verificámos que parece existir uma maior isodensidade dos oncocitomas em relação em parênquima renal normal. Em média, a diferença de contraste entre os oncocitomas e o córtex renal foi de $43,5 \mathrm{HU}$, enquanto para os CCR foi de 59,7 HU. Utilizámos estes dados para tentar determinar um cut-off a partir do qual seria mais provável diagnosticarmos um oncocitoma em vez de um CCR-cC e verificámos que, para um diferencial (HU córtex-HU tumor) $<40$, a probabilidade de diagnosticarmos um oncocitoma era de $50 \%$ e apenas de $25 \%$ para um CCR-cc. Também constatámos que quanto menor a diferença de atenuação $(\mathrm{HU})$ entre o córtex e o tumor renal suspeito, maior a probabilidade de se tratar de um oncocitoma.

Neste âmbito, também têm surgido alguns estudos como o de Gakis et al. que demonstraram existir diferenças nos padrões de captação de contraste entre os oncocitomas e CCRs-cc, na TC multifásica, em particular na fase córtico-medular (avaliada aos 35-40 segundos após a administração de contraste $)^{16}$. No entanto, à semelhança do nosso trabalho, a amostra é pequena e são necessários mais estudos que corroborem estes resultados. Por outro lado, também encontramos na literatura estudos como o de Choudhary et al., onde são perentórios na afirmação de que a TC não consegue distinguir o oncocitoma de outros tumores sólidos renais ${ }^{17}$, o que revela a controvérsia do tema.

Atualmente, a abordagem das PMR centra-se principalmente na nefrectomia poupadora de nefrónios ${ }^{18}$. No entanto, se tivermos em conta a incidência não negligenciável de oncocitomas (tumores benignos, sem potencial metastático) nas PMR (até 30\%), o diagnóstico incidental de PMR em idades cada vez mais avançadas ( $>75$ anos), em doentes com múltiplas comorbilidades e sem condições operatórias, de forma a evitar o sobretratamento, faz sentido começarmos a estabelecer protocolos de avaliação por TC que nos permitam orientar de forma mais fundamentada a nossa decisão terapêutica. Logo, determinarmos o diferencial de contraste entre o córtex renal e o tumor permitir-nos-á decidir quais os doentes que terão, por exemplo, indicação para a realização de uma biopsia percutânea do tumor renal, um procedimento que evoluiu ao longo dos últimos anos, sendo considerado atualmente um procedimento eficaz e seguro ${ }^{19}$.

Reconhecemos as limitações deste estudo relacionadas com o número reduzido da amostra, a sua natureza retrospetiva, tal como a exclusão de CCR de outras histologias, como 

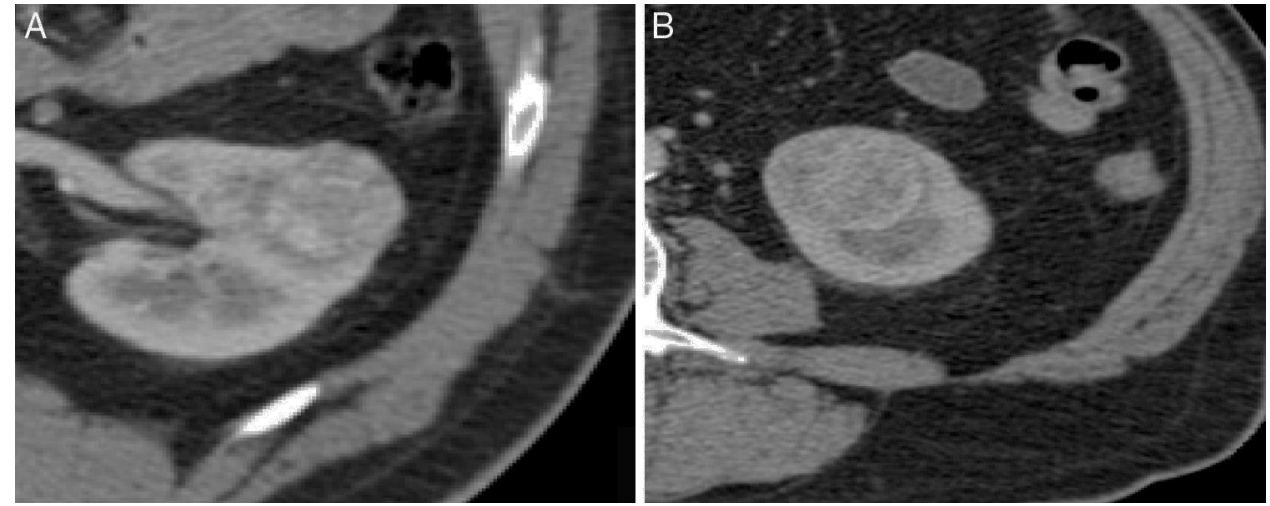

Figura 3 À esquerda, temos um oncocitoma. À direita, um CCR-cc. Não existem diferenças morfológicas distintivas, bem como a captação de contraste foi aproximadamente idêntica: $49 \mathrm{HU}$ e $51 \mathrm{HU}$, respetivamente.

os tipos papilar e cromófobo. No entanto, salvaguardamos o facto de, atualmente, ainda não existirem protocolos de diagnóstico imagiológico de oncocitomas num contexto pré-operatório, logo, as características funcionais da TC devem ser exploradas ao seu máximo de eficiência.

\section{Conclusão}

$\mathrm{Na}$ avaliação imagiológica por URO-TC, nomeadamente na fase nefrográfica, parece existir uma tendência para maior isodensidade dos oncocitomas em relação ao parênquima renal normal. Este achado poderá contribuir para uma melhor decisão terapêutica, na medida em que nos poderá direcionar para uma biopsia de confirmação em detrimento da excisão cirúrgica.

\section{Responsabilidades éticas}

Proteção de pessoas e animais. Os autores declaram que para esta investigação não se realizaram experiências em seres humanos e/ou animais.

Confidencialidade dos dados. Os autores declaram que não aparecem dados de pacientes neste artigo.

Direito à privacidade e consentimento escrito. Os autores declaram que não aparecem dados de pacientes neste artigo.

\section{Financiamento}

Este trabalho foi realizado sem patrocínio ou apoio financeiro.

\section{Conflito de interesses}

Os autores declaram não haver conflito de interesses.

\section{Bibliografia}

1. Lane BR, Tolbert CM, Kiesinger CB. Growth kinetics and active surveillance for small renal masses. Curr Opin Urol. 2012;22:353-9.

2. Volpe A, Panzarella T, Rendon RA, Haider MA, Kondylis FI, Jewett MA. The natural history of incidentally detected small renal masses. Cancer. 2004;100:738-45.

3. Frank T, Blute ML, Cheville JC, Lohse CM, Weaver AL, Zincke H. Solid renal tumors: An analysis of pathological features related to tumor size. J Urol. 2003;170:2217-20.

4. Soga N, Nishikawa K, Takaki H, Yamada Y, Arima K, Hayashi N, et al. Low incidence of benign lesions in resected suspicious renal masses greater than $2 \mathrm{~cm}$ : Single-center experience from Japan. Int J Urol. 2012;19:729-34.

5. Schlomer B, Figenshau RS, Yan Y, Venkatesh R, Bhayani SB. Pathological features of renal neoplasms classified by size and symptomatology. J Urol. 2006;176:1317-20.

6. Duchene DA, Lotan Y, Cadeddu JA, Sagalowsky Al, Koeneman KS. Histopathology of surgically managed renal tumors: Analysis of a contemporary series. Urology. 2003;62:827-30.

7. Violette P, Abourbih S, Szymanski KM, Tanguay S, Aprikian A, Matthews K, et al. Solitary solid renal mass: Can we predict malignancy? BJU Int. 2012;110:548-52.

8. Ehsani L, Seth R, Bacopulos S, Seth A, Osunkoya AO. BCA2 is differentially expressed in renal oncocytoma an analysis of 158 renal neoplasms. Tumour Biol. 2013;34(2):787-91.

9. Perez-Ordonez B, Hamed G, Campbell S, Erlandson RA, Russo P, Gaudin PB, et al. Renal oncocytoma: A clinicopathologic study of 70 cases. Am J Surg Pathol. 1997;21(8):871-83.

10. Garant M, Bonaldi VM, Taourel P, Pinsky MF, Bret PM. Enhancement patterns of renal masses during multiphase helical CT acquisitions. Abdom Imaging. 1998;23(4):431-6.

11. Neisius D, Braedel HU, Schindler E, Hoene E, Alloussi S. Computed tomographic and angiographic findings in renal oncocytoma. Br J Radiol. 1988;61(731):1019-25.

12. Millet I, Doyon FC, Hoa D, Thuret R, Merigeaud S, Serre I, et al. Characterization of small solid renal lesions: Can benign and malignant tumors be differentiated with CT? AJR Am J Roentgenol. 2011;197(4):887-96.

13. Cornelis F, Tricaud E, Lasserre AS, Petitpierre F, Bernhard JC, le Bras $Y$, et al. Routinely performed multiparametric magnetic resonance imaging helps to differentiate common subtypes of renal tumours. Eur Radiol. 2014;24(5):1068-80.

14. Wu J, Zhu Q, Zhu W, Chen W, Wang S. Comparative study of CT appearances in renal oncocytoma and chromophobe renal cell carcinoma. Acta Radiol. 2015. 
15. Zhang J, Lefkowitz RA, Ishill NM, Wang L, Moskowitz CS, Russo P, et al. Solid renal cortical tumors: Differentiation with CT. Radiology. 2007 Aug;244(2):494-504.

16. Gakis G, Kramer U, Schilling D, Kruck S, Stenzl A, Schlemmer HP. Small renal oncocytomas: Differentiation with multiphase CT. Eur J Radiol. 2011 Nov;80(2):274-8.

17. Choudhary S, Rajesh A, Mayer NJ, Mulcahy KA, Haroon A. Renal oncocytoma: CT features cannot reliably distinguish oncocytoma from other renal neoplasms. Clin Radiol. 2009 May;64(5):517-22.

18. EAU guidelines-Renal cell carcinoma 2015.

19. Richard PO, Jewett MA, Bhatt JR, Kachura JR, Evans AJ, Zlotta AR, et al. Tumor biopsy for small renal masses: A single-center 13-year experience. Eur Urol. 2015 Dec;68(6): 1007-13. 